Published in final edited form as:

Clin Liver Dis. 2013 November ; 17(4): . doi:10.1016/j.cld.2013.07.001.

\title{
Drug-induced Acute Liver Failure
}

\author{
William M. Lee, MD \\ UT Southwestern Medical Center at Dallas, Dallas, TX, USA William.Lee@utsouthwestern.edu
}

\author{
Keywords \\ Hepatocyte injury; Encephalopathy; Coagulopathy; Liver transplantation
}

\section{BACKGROUND}

Acute liver failure (ALF), where loss of hepatocyte function occurs over days or weeks without evidence of cirrhosis, has traditionally been defined by altered mentation accompanied by coagulopathy (prolonged international normalized ratio [INR]). Loss of nearly the entire hepatocyte mass is observed in this setting because of a variety of agents including viruses, toxins, and drugs. Recovery depends on whether the injury is ongoing or self-limited and whether or not hepatocytes are capable of regenerating. It is estimated that approximately 2000 people experience ALF annually in the United States and nearly $60 \%$ of these are caused by acetaminophen or idiosyncratic drug reactions, drug-induced liver injury (DILI) in its largest sense. Overall, acetaminophen injury far exceeds idiosyncratic DILI by 4:1 among cases reaching the threshold of ALF. Idiosyncratic reactions to prescription drugs or complementary and alternative medications (CAMS) are referred to as DILI, whereas acetaminophen hepatotoxicity is often referred to separately under the acronym APAP. Over the past 16 years, APAP-related toxicity leading to ALF has comprised $46 \%$ of subjects enrolled in the Acute Liver Failure Study Group (ALFSG), a network that currently includes 16 transplant centers across the United States. ${ }^{1}$ By contrast, DILI-related ALF was observed in $11 \%$ of these same subjects. Still, DILI is the largest single group after APAP (Fig. 1).

This was not the case before the 1990s when hepatitis B was more prevalent and acetaminophen was not as widely used. Early studies did not mention acetaminophen-related injury; nearly 50\% were thought related to acute hepatitis B. Even in the mid-1990s only $20 \%$ of cases were determined to be caused by APAP overdoses. ${ }^{2}$

DILI caused by either idiosyncratic reactions or acetaminophen-related hepatic necrosis leads to ALF on very different trajectories. A prescription drug, such as isoniazid, leads to a subacute pattern where injury evolves gradually over several weeks, and encephalopathy occurs generally after a minimum of 2 to 4 weeks from onset of illness. By contrast, acetaminophen-related injury is termed hyperacute rather than subacute and its evolution is measured in hours, with peak injury occurring about 72 hours after a single time point ingestion of a toxic amount (Table 1). Although patients with ALF may exhibit these features, patients with APAP more commonly exhibit advanced degrees of hepatic coma on admission to transplant centers, sometimes having signs of cerebral edema that results in uncal herniation and compression of the brainstem that is nearly uniformly fatal.

Fortunately, only between $10 \%$ and $20 \%$ of all patients with ALF show evidence of cerebral edema and its incidence may be declining. ${ }^{3}$ 


\section{ACETAMINOPHEN-RELATED ALF}

Acetaminophen-related ALF was traditionally recognized as caused by suicidal overdoses, although frequent mention was made in the 1980s of therapeutic misadventures or the "alcohol-Tylenol" syndrome. ${ }^{4,5}$ Thus, most cases can be divided into intentional and unintentional cases. Intentional cases represent a single time point ingestion for self-harm, whereas unintentional cases are defined as ingestions in excess of package labeling instructions, occurring over several days (or weeks) with pain (usually chronic back or pancreatic pain, but also postsurgical pain and other miscellaneous causes). ${ }^{6}$ The overall burden of intentional cases greatly exceeds accidental ones in terms of emergency room visits where all patients once recognized should immediately receive the antidote, $N$ acetylcysteine (NAC; obtained in intravenous form as Acetadote). Among those reaching the threshold of ALF, there are nearly equal numbers of intentional and unintentional cases. Because APAP-related ALF has become commonplace, instances of APAP-related injury should be readily recognized. However, many are already comatose on arrival to the tertiary care facility and the ingestion history may be lacking. Similarly, the use of acetaminophen levels in patients already demonstrating massive hepatocyte injury may create false impressions that the cause is not acetaminophen. Thus, a high index of suspicion is needed to recognize all cases. Recently, it has become apparent that the biochemical profile itself can be a strong indication for acetaminophen hepatotoxicity: the finding of remarkably high aminotransferases $(>3500 \mathrm{IU} / \mathrm{L})$ in concert with low bilirubin levels $(<5)$ is the nearly unique signature of APAP injury. ${ }^{7}$ Typical aminotransferase levels exceed 10,000 IU/L. Ischemic hepatopathy and rare instances of hepatitis B or herpes simplex hepatitis are the only alternate diagnoses that should be entertained. A point-of-care assay to detect acetaminophen adducts, the by-product of cell injury rather than the parent compound, currently is being developed. Acetaminophen hepatic injury is covered in more detail elsewhere in this issue.

\section{IDIOSYNCRATIC DRUG-RELATED LIVER INJURY}

Most reactions to prescription drugs or CAMs are considered idiosyncratic; they are unpredictable, vary greatly in severity, and occur at varying time intervals after ingestion (anywhere from a few days to 1 year). ${ }^{8}$ Their toxicity is usually unrelated to dose, route, or duration of drug administration (although a review of drugs withdrawn from the market in the United States recently found that most were prescribed at daily doses $>50 \mathrm{mg}$ per day). ${ }^{9}$ Instead, the Greek root of idiosyncrasy emphasizes that it is the unique characteristics of the individual that predispose them to develop a toxic reaction, whereas many others can use the drug safely. ${ }^{10}$ It is assumed that the adverse reactions to these xenobiotic compounds have a strong genetic basis. ${ }^{11}$ DILI, as a result, has become an important catalyst for pharmacogenomics research. ${ }^{12}$ Only a fraction of DILI reactions lead to ALF. Among the first 300 patients enrolled in the National Institutes of Health-supported Drug-Induced Liver Injury Network (DILIN), 33\% were hospitalized, 15\% were considered severe, and 6\% died or underwent transplantation. ${ }^{13}$ Thus, only a small fraction of the overall group experienced ALF, although there were $9 \%$ fatalities or transplants in the subgroup where hepatocellular injury was present. Thus, the spectrum of types and severity of liver injury caused by DILI overall is broad and expectation for recovery from the average instance of DILI is the rule.

The phenotype associated with ALF is characterized by hepatocellular injury that typically evolves more slowly than acetaminophen and is remarkably rare, with some agents in the range of 1:30,000 to 100,000 prescriptions. A wide variety of medications have been implicated, although not every one can cause these severe forms of liver damage. Antibiotics, anticonvulsants, and nonsteroidal anti-inflammatory agents are among the most commonly implicated medications, with isoniazid continuing its prominent place among 
those frequently implicated. The ALFSG review of 133 patients enrolled in the registry with ALF determined that antimicrobials were the most common cause for ALF accounting for

$46 \%$ of cases. ${ }^{14}$ Box 1 lists a current update of commonly seen drugs modified from Reuben and colleagues. ${ }^{14}$ Over time the list of agents implicated evolves, because usage changes as new agents come along. For example, phenytoin is used much less currently than it was in an earlier era. Thus, a decline in phenytoin ALF should be seen. Telithromycin (Ketek) was a new antibiotic that gained in popularity as a replacement for macrolide antibiotics because its spectrum of activity included pneumococci resistant to penicillins and even macrolides; however, the identification of several instances of ALF in the 3 years after its approval has led to revised approval with much more limited indications and a black box warning from the Food and Drug Administration. ${ }^{15,16}$ It is hoped that isoniazid will lose its primacy as the keystone agent against tuberculosis, but to date that has not happened.

In the paper cited previously that reviewed the experience of 133 ALF DILI cases, spontaneous recovery was limited, although the slower evolution of disease often allowed for transplantation to take place. Transplant-free survival (TFS) was $27 \%$, whereas $42 \%$ received a graft, yielding an overall short-term survival of $66 \%$. A recent update of these figures from ALFSG for 220 DILI cases indicated that TFS is even worse, at 23\%, with $40 \%$ transplanted giving an overall survival of only $58 \%$ (unpublished data). By comparison, acetaminophen short-term results for 916 individuals admitted with ALF included 63\% TFS, 9\% transplanted, giving a 70\% overall short-term survival. Fewer APAP cases are candidates for liver grafting because of ongoing substance abuse or lack of insurance funding, but they less frequently seem to require rescue. It is postulated that the self-limited duration of injury, despite its severity, allows rapid regeneration and resolution of the hepatic damage. Pathogenesis of the two forms of DILI (APAP and idiosyncratic DILI) remains a black box. ${ }^{17}$

Clinical recognition of DILI ALF is little different from that of APAP except for the slower speed of disease evolution. Any patient in the emergency room showing signs of jaundice and coagulopathy with or without altered mentation should undergo further screening to determine if ALF is present. All patients should receive careful questioning regarding recent medications, CAMs, and any use of acetaminophen-containing compounds and an extensive serologic work-up focusing on determining the cause of the condition. Those with subacute injury of whatever cause may not exhibit significant encephalopathy early on, but linger with modest aminotransferase elevations but elevated INR and bilirubin levels that predict a poor outcome once they persist for more than 10 to 14 days. These patients are more likely to develop ascites, infection, and renal insufficiency. Cause of death in the absence of transplantation is primarily systemic infection or cerebral edema.

\section{OVERALL MANAGEMENT}

Intensive care management is used for virtually all patients with any degree of encephalopathy and can be considered for those with profound coagulopathy where evolution to altered mentation is likely. Extensive guidelines have been established for management, although much is based on expert opinion rather than evidence-based medicine, because large controlled trials are seldom possible, given the orphan status of this syndrome. ${ }^{18,19}$ In general, patients are placed with head elevated and receive frequent checks for neurologic function. Most are dehydrated on arrival and require volume resuscitation. Initial serologic testing is used to exclude other causes, even if a putative drug cause has been elicited. In a recent study from the DILIN, 9 out of 318 cases tested for hepatitis $\mathrm{E}$ were recategorized as having likely hepatitis $\mathrm{E}$ based on the presence of IgM anti-hepatitis E antibody, and in four patients also hepatitis E virus RNA. ${ }^{20}$ Only one of these cases manifested signs of ALF. Thus, casting a wide initial testing net for causes and, 
in particular, repeat questioning of the patient or their family concerning use of antibiotics even remotely or herbal or dietary supplements is remarkably helpful.

There is no certain antidote for ALF at this time. Specific measures are available for acetaminophen (NAC). Although NAC use after onset of encephalopathy theoretically may help prevent worsening, it is being given too late to prevent the unfolding injury. With regard to idiosyncratic DILI, many patients continue to consume the suspect agent after onset of symptoms and this, in general, is harmful, although no study to date has shown with certainty that early withdrawal improves outcomes. In some instances, a drug taken only for 2 to 3 days may lead to a fatal outcome.

\section{Treatment with NAC}

NAC is the recognized antidote for acetaminophen poisoning and long has been claimed to be effective in nonacetaminophen settings, without much supportive evidence. NAC was subjected to a randomized placebo-controlled trial for nonacetaminophen ALF that included DILI as one subgroup. The trial included 173 randomized patients, the largest ever performed in ALF. The group consisted of four causes: (1) DILI; (2) autoimmune hepatitis; (3) hepatitis B; and (4) indeterminate, those where a cause could not be discerned. The primary outcome (improvement in overall survival) was not achieved but significant improvement was observed within early coma grade patients (I to II): SS 52\% with NAC versus $30 \%$ with placebo. ${ }^{21}$ All ALF trials in the modern era are compromised by transplantation that rescues approximately $40 \%$ of those with non-APAP ALF so that their true outcome will never be known and overall survival is improved because of the use of liver grafting, as it should be. Because short-term survival after transplantation is more than $90 \%$ and mainly hinges on technical issues, such as graft quality, the impact of an intravenous medication is unlikely to affect overall outcome particularly in those with advanced hepatic failure, where death or transplant as outcomes occur quickly. We observed that $50 \%$ of coma III to IV patients achieved an outcome (death or transplant) by Day 4, whereas those with lesser coma grades only reached this threshold at Day 10 after admission to study. Thus, in retrospect, TFS is probably a better outcome measure than overall survival. It seems unlikely that patients with advanced coma grades would benefit so rapidly from NAC that they would not progress to an outcome as described. There were no safety issues and, using a Cox proportional hazards model, outcomes were better for the NAC Coma I to II group than all other categories (Fig. 2). The percent transplanted was significantly lower in the NAC group when follow-up extended out to 1 year. There was a trend toward shorter intensive care unit and hospital stays in favor of the NAC group, but this was not significant. The greatest improvement in TFS was seen in those treated with NAC (Table 2). For those with etiology as DILI TFS was 58\% for NAC versus $27 \%$ for placebo, and for hepatitis B $40 \%$ for NAC versus $17 \%$ for placebo.

Two subsequent studies have focused on why NAC might have made a difference. Stravitz and colleagues measured levels at two time points of 10 cytokines, using a multiplex enzyme-linked immunosorbent assay on sera from 78 patients who participated in the NAC trial (unpublished data). Treatment with NAC and low interleukin (IL)-17 levels were associated with improved outcomes in multivariate analysis. Among those with elevated IL-17 levels, $78 \%$ of the NAC-treated patients and $44 \%$ of the control subjects had undetectable IL-17 levels on Day 3 after admission to study. Thus, NAC may act by lowering IL-17 levels, thus averting ongoing liver damage at this crucial disease stage. In a second study, reviewing standard biochemistries from the NAC trial, Singh and colleagues ${ }^{22}$ showed that patients with early coma grade who were treated with NAC showed significant improvement in bilirubin and alanine aminotransferase levels compared with the other three groups (maximum $P<.02$ for NAC $1-2$ vs the three other treatments) when predicting death or transplantation. These two studies support the use of NAC in early stage hepatic coma 
caused by drug-related liver injury. To date, the Food and Drug Administration has not approved NAC for the indication of nonacetaminophen ALF.

\section{Corticosteroid Use}

Corticosteroid therapy has been proposed as treatment of DILI in the ALF setting but little evidence advanced to support it, and unlike alcoholic hepatitis, no controlled trials have been performed. ${ }^{23}$ A recent analysis of the ALFSG database sought to provide circumstantial evidence for or against steroid use by reviewing outcomes for those who did and did not receive corticosteroid therapy for their ALF, regardless of cause (unpublished data). Of 131 patients with DILI ALF, 26 had received corticosteroids and 105 had not as shown in records examined. Survival was 50\% for those receiving and $62 \%$ for those not receiving steroid therapy. This was uncontrolled and a bias toward treatment of desperately sick individuals may have been at play here.

\section{Hepatic Encephalopathy}

Understanding the encephalopathy that occurs in ALF has been limited by the relative rarity of the condition. The role of ammonia as a causative agent has been debated. High ammonia levels correlate in a general way with the degree of encephalopathy but not that well, but do correlate more closely to the presence of cerebral edema. Acute kidney injury occurs in $70 \%$ of patients with acetaminophen-induced hepatic necrosis and encephalopathy. Recent data from ALFSG has established that high ammonia levels are virtually never seen in the absence of acute kidney injury. Hyperacute liver injury is closely associated with cerebral edema and this has been presumed to be related to the acuity of the injury; however, the most common cause of hyperacute ALF is acetaminophen and the presence of renal injury clearly plays a part in causing the high ammonia levels.

Use of lactulose and antibiotics in patients with ALF has been problematic, because colonic distention caused by lactulose is counterproductive in a surgical setting. ${ }^{18}$ No proof of efficacy has been shown for either form of management classically used in encephalopathy caused by cirrhosis.

ALFSG is currently testing ornithine phenylacetate as an ammonia-trapping agent in an initial pilot study to evaluate safety and tolerability. Ammonia-lowering agents could represent a significant improvement in encephalopathy management and would demonstrate once and for all whether ammonia directly contributes to this condition. ${ }^{24,25}$

\section{TRANSPLANTATION AND PROGNOSIS}

Transplantation provides a reliable rescue for patients when no signs of recovery are forthcoming. Only a small fraction of patients receive a liver graft: $24 \%$ of all patients with ALF, $9 \%$ of acetaminophen ALF, and $40 \%$ of patients with DILI ALF. For each patient, a careful assessment of need and appropriateness precedes listing so a prognostic score has long been considered a vital but elusive goal. Even when a patient is listed, organ availability determines ultimate outcome and patient candidacy can change from good to poor over hours. A review of transplantation data from the United Network for Organ Sharing registry disclosed that among patients undergoing liver transplantation between 1990 and 2002, a total of 2291 were considered to have acute hepatic necrosis, which is close to but may not be identical with ALF as described previously. ${ }^{26}$ Of these, 357 (15\%) were thought caused by DILI but including APAP. Thus, roughly half received a graft for APAP, the other half for idiosyncratic DILI. This suggests that despite the high morbidity of DILI once the threshold of ALF is reached, the number of patients actually undergoing transplantation for DILI remains small. It is important to point out that the United Network for Organ Sharing database only registers those listed for transplantation and receiving a 
graft, so this is a skewed group compared with the larger overall universe of patients with DILI that is more uniformly captured by ALFSG or DILIN. Once again, ALFSG only identifies those DILI cases meeting criteria of hepatic encephalopathy and prolonged INR, only $11 \%$ of all ALFSG enrolled patients and 9\% of the all DILI patients (according to DILIN data). ${ }^{13,14}$ Among ALFSG patients as noted, the slower evolution of disease and higher fatality rate or failure to improve has yielded a higher percentage of transplants undertaken in this group compared with those with acetaminophen-related hepatic failure. The higher number of acetaminophen cases results in similar total numbers of patients being transplanted for APAP and DILI.

When outcomes for the ALFSG group of 133 patients with DILI reviewed previously were examined, the Model for End Stage Liver Disease (MELD) score and coma grade on admission were the strongest predictors of liver transplantation. ${ }^{14}$ Because most of these patients would have received a listing as Status 1, the highest priority, it is not clear whether MELD is relevant here. To date, robust prognostic scoring systems have not proved useful in ALF. Although Kings College Hospital (KCH) criteria have been used by some centers, it is evident that those reaching the threshold of Kings criteria are highly likely to require transplantation; those not reaching criteria still have a 50\% mortality, indicating the limits of such a scoring system. ${ }^{26,27}$ Other more global scores that reflect intensive care unit survival have not proved to be of any additional value. Cholongitas and colleagues ${ }^{28}$ studied 125 consecutive patients with APAP, comparing Sequential Organ Failure Assessment (SOFA), MELD, Acute Physiologic and Chronic Health Assessment II (APACHE II), and KCH. They concluded that SOFA performed best of the four with an area under the receiver operator curve (AUC) of 0.79, whereas the AUCs for MELD, APACHE II, and KCH were $0.58,0.72$, and 0.65 , respectively. This study applied only to APAP cases, suggesting that it may be necessary to develop a prognostic score for each ALF etiology. Taylor and colleagues ${ }^{29}$ have developed a prognostic score with great accuracy for hepatitis A. More recently, an index was developed that outperformed MELD and KCH across the spectrum of ALF conditions. ${ }^{30}$ In this system, M-30 antigen, a marker of apoptosis, was used in concert with coma grade, INR, bilirubin, and phosphorus and gave an AUC of 0.822 compared with MELD at 0.704 and $\mathrm{KCH}$ at 0.654 . A direct comparison with SOFA was not made. The limitation of this index is that it requires a special test with limited availability to complete the index. M-30 did contribute to improving to the final score. Similarly, a study from Germany ${ }^{31}$ substituted M-65 antigen levels for bilirubin in the MELD score to determine survival or need for transplantation or death in a wide spectrum of ALF where APAP constitute $13 \%$ and DILI 18\%. In this study, the AUC for the modified MELD, referred to as M-MELD, was 0.87 on admission and better if maximum M- 65 values were used; however, as a retrospective study one only knows what the maximum value obtained is after the study is completed. Similarly, the real value of a prognostic score must be on admission, when the decision to list is paramount. Outcomes later will become clarified and all prognostic scores will tend to improve. Further studies are under way to nail down a robust score that is applicable (or modified) for all etiologic groups and could be simplified to be developed as an "app," rendering it more user-friendly than current scores.

\section{GENOMICS AND THE FUTURE}

Studies have tried to determine whether there is a specific host genomics signature that identifies increased susceptibility to catastrophic liver injury, because the ALF group of patients with DILI represents the tip of a much larger iceberg. For every INH ALF patient, there are probably nine additional patients with alanine aminotransferase elevations not requiring and this was the original message in Hy law. ${ }^{32}$ This is across a variety of drug groups and types. The question becomes: What makes one or another patient have a poor outcome with seemingly similar clinical features in other respects? Specific HLA haplotypes 
have been associated with certain drugs in genome-wide association studies. ${ }^{11,12}$ However, a recent study analyzed whether there were keratin 8 and 18 host mutations that are considered to be cytoprotective that would possibly be underrepresented among a cohort of 344 patients with ALF. Indeed, keratin 8 variants in particular were overrepresented in white patients with ALF across etiologies. ${ }^{33}$ This provides support for the concept that host increased susceptibility underlies conversion to ALF, although it is likely that this susceptibility is multifactorial. A further study has also suggested a specific acetaminophen UDP-glucuronosyltransferase enzyme polymorphism can explain the variable susceptibility of individuals to acetaminophen toxicity. ${ }^{34}$

Overall, it seems that genomic studies in the future will help identify those at greatest risk of unfavorable outcomes. Whether these assays turn out to be specific to drugs or are more global remains to be seen.

\section{SUMMARY}

Although ALF caused by DILI comprises a small fraction of overall DILI, these patients require high resource use and have relatively poor outcomes. DILI caused by idiosyncrasy more often leads to death or transplantation than does acetaminophen ALF, but the number of patients in each category receiving a graft is roughly the same. Efforts to improve outcomes should focus on more effective treatments and better methods to identify those that might experience poor outcomes.

\section{REFERENCES}

1. Ostapowicz GA, Fontana RJ, Schiodt FV, et al. Results of a prospective study of acute liver failure at 17 tertiary care centers in the United States. Ann Intern Med. 2002; 137:945-54.

2. Schiødt FV, Atillasoy E, Shakil O, et al. Etiology and outcome for 295 patients with acute liver failure in the United States. Liver Transpl Surg. 1999; 5:29-34. [PubMed: 9873089]

3. Bernal W, Auzinger G, Dhawan A, et al. Acute liver failure. Lancet. 2010; 376:190-201. [PubMed: 20638564]

4. Zimmerman HJ, Maddrey WC. Acetaminophen (paracetamol) hepatotoxicity with regular intake of alcohol: analysis of instances of therapeutic misadventure. Hepatology. 1995; 22:767-73. [PubMed: 7657281]

5. Wootton FT, Lee WM. Acetaminophen hepatotoxicity in the alcoholic. South Med J. 1990; 83:1047-9. [PubMed: 2402650]

6. Schiødt FV, Rochling FJ, Casey DL, et al. Acetaminophen toxicity in an urban county hospital. N Engl J Med. 1997; 337:1112-7. [PubMed: 9329933]

7. Khandelwal N, James LP, Sanders C, et al. Acute Liver Failure Study Group. Unrecognized acetaminophen toxicity as a cause of indeterminate acute liver failure. Hepatology. 2011; 53:56776. [PubMed: 21274877]

8. Kaplowitz N. Idiosyncratic drug hepatotoxicity. Nat Rev Drug Discov. 2005; 4:489-99. [PubMed: 15931258]

9. Tujios S, Fontana RJ. Mechanisms of drug-induced liver injury: from bedside to bench. Nat Rev Gastroenterol Hepatol. 2011; 8:202-11. [PubMed: 21386809]

10. Lee WM, Senior JR. Recognizing drug-induced liver injury: current problems, possible solutions. Toxicol Pathol. 2005; 33:155-64. [PubMed: 15805067]

11. Daly AK, Donaldson PT, Bhatnagar P, et al. HLA-B*5701 genotype is a major determinant of drug-induced liver injury due to flucloxacillin. Nat Genet. 2009; 41:816-9. [PubMed: 19483685]

12. Urban TJ. Whole-genome sequencing in pharmacogenetics. Pharmacogenomics. 2013; 14:345-8. [PubMed: 23438878]

13. Chalasani N, Fontana RJ, Bonkovsky HL, et al. Causes, clinical features, and outcomes from a prospective study of drug induced liver injury in the United States. Gastroenterology. 2008; 135:1924-34. [PubMed: 18955056] 
14. Reuben A, Koch DG, Lee WM. Drug-induced acute liver failure: results of a U.S. multicenter, prospective study. Hepatology. 2010; 52:2065-76. [PubMed: 20949552]

15. Brinker AD, Wassel RT, Lyndly J, et al. Telithromycin-associated hepatotoxicity: clinical spectrum and causality assessment of 42 cases. Hepatology. 2009; 49:250-7. [PubMed: 19085949]

16. Soreth J, Cox E, Kweder J, et al. Ketek: the FDA perspective. N Engl J Med. 2007; 356:1675-6. [PubMed: 17442912]

17. Jones DP, Lemasters JJ, Han D, et al. Mechanisms of pathogenesis in drug hepatotoxicity putting the stress on mitochondria. Mol Interv. 2010; 10:98-111. [PubMed: 20368370]

18. Stravitz RT, Kramer AH, Davern T, et al. Acute Liver Failure Study Group. Intensive care of patients with acute liver failure: recommendations of the Acute Liver Failure Study Group. Crit Care Med. 2007; 35:2498-508. [PubMed: 17901832]

19. Lee WM, Stravitz RT, Larson AM. Introduction to the revised American Association for the Study of Liver Diseases Position Paper on acute liver failure 2011. Hepatology. 2012; 55:965-7. [PubMed: 22213561]

20. Davern TJ, Chalasani N, Fontana R, et al. Acute hepatitis E infection accounts for some cases of suspected drug-induced liver injury. Gastroenterology. 2011; 141:1665-72. [PubMed: 21855518]

21. Lee WM, Hynan LS, Rossaro L, et al. Intravenous N-acetylcysteine improves transplant-free survival in early stage non-acetaminophen acute liver failure. Gastroenterology. 2009; 137:85664. [PubMed: 19524577]

22. Singh S, Hynan LS, Lee WM, Acute Liver Failure Study Group. Improvements in hepatic serological biomarkers are associated with clinical benefit of intravenous $\mathrm{N}$-acetylcysteine in early stage non-acetaminophen acute liver failure. Dig Dis Sci. 2013; 58(5):1397-402. [PubMed: 23325162]

23. Ichai P, Duclos-Vallée J, Guettier C, et al. Usefulness of corticosteroids for the treatment of severe and fulminant forms of autoimmune hepatitis. Liver Transpl. 2007; 13:996-1003. [PubMed: 17370335]

24. Jalan R, Wright G, Davies NA, et al. L-Ornithine phenylacetate (OP): a novel treatment for hyperammonemia and hepatic encephalopathy. Med Hypotheses. 2007; 69:1064-9. [PubMed: 17467190]

25. Lee WM, Jalan RV. Treatment of hyperammonemia in liver failure: a tale of two enzymes. Gastroenterology. 2009; 136:2048-51. [PubMed: 19409290]

26. Larson AM, Fontana RJ, Davern TJ, et al. Acute Liver Failure Study Group. Acetaminopheninduced acute liver failure: results of a United States multicenter, prospective study. Hepatology. 2005; 42:1367-72.

27. Russo M, Galanko JA, Shrestha R, et al. Liver transplantation for acute liver failure from drug induced liver injury in the United States. Liver Transpl. 2004; 10:1018-23. [PubMed: 15390328]

28. Cholongitas E, Theocharidou E, Vasianopoulou P, et al. Comparison of the sequential organ failure assessment score with the king's college hospital criteria and the model for end-stage liver disease score for the prognosis of acetaminophen-induced acute liver failure. Liver Transpl. 2012; 18:40512. [PubMed: 22213443]

29. Taylor RM, Davern TJ, Munoz S, et al. Acute Liver Failure Study Group. Fulminant hepatitis A virus infection in the United States: incidence, prognosis, and outcomes. Hepatology. 2006; 44:1589-97. [PubMed: 17133489]

30. Rutherford A, King LY, Hynan LS, et al. Acute Liver Failure Study Group. Development of an accurate index for predicting outcomes of patients with acute liver failure. Gastroenterology. 2012; 143:1237-43. [PubMed: 22885329]

31. Bechmann LP, Jochum C, Kocabayoglu P, et al. Cytokeratin 18-based modification of the MELD score improves prediction of spontaneous survival after acute liver injury. J Hepatol. 2010; 53:639-47. [PubMed: 20630612]

32. Temple R. Hy's law: predicting serious hepatotoxicity. Pharmacoepidemiol Drug Saf. 2006; 15:241-3. [PubMed: 16552790]

33. Strnad P, Zhou Q, Hanada S, et al. Acute Liver Failure Study Group. Ethnic-specific keratin variants predispose to an adverse outcome in acute liver failure. Gastroenterology. 2010; 139:82835. [PubMed: 20538000] 
34. Court MH, Freytsis M, Wang X, et al. The UDP-Glucuronosyltransferase (UGT) 1A polymorphism c.2042C $>\mathrm{G}$ (rs8330) is associated with increased human liver acetaminophen glucuronidation, increased UGT1A Exon 5a/5b splice variant mRNA ratio, and decreased risk of unintentional acetaminophen-induced acute liver failure. J Pharmacol Exp Ther. 2013; 345:297307. [PubMed: 23408116] 


\section{Box 1}

\section{Drugs implicated in causing acute liver failure}

Antimicrobials

Isoniazid, with or without rifampicin

Trimethoprim/sulfamethoxazole

Nitrofurantoin

Terbinafine

Itraconazole

Ketoconazole

Amoxicillin

Ciprofloxacin

Azithromycin

Telithromycin

Anticonvulsants

Phenytoin

Valproic acid

Carbamazepine

Complementary and alternative medications

Lipokinetix (usnic acid)

Herbalife (unspecified)

Ma-huang and other Chinese herbs

Hydroxycut (unspecified)

Others

Diclofenac

Etodolac

Disulfiram

Propylthiouracil

Methyldopa

Chemotherapeutic agents

Gemtuzumab

Adapted from Reuben A, Koch DG, Lee WM. Drug-induced acute liver failure: results of a U.S. multicenter, prospective study. Hepatology 2010;52:2065-76. 


\section{KEY POINTS}

- Acute liver failure, the most severe form of liver injury, is comprised primarily of instances of drug-induced liver injury (DILI).

- Although acetaminophen overdoses dominate, a significant portion of DILI is related to prescription drugs and complementary and alternative medications.

- Acetaminophen and idiosyncratic cases differ dramatically in clinical and biochemical features and in outcomes.

- Therapy for these conditions has been limited to date, although $N$-acetylcysteine may be of benefit.

- Survival without transplantation is particularly poor for those with idiosyncratic DILI.

- Efforts to improve outcomes should focus on pathogenesis and improving hepatocyte regeneration. 


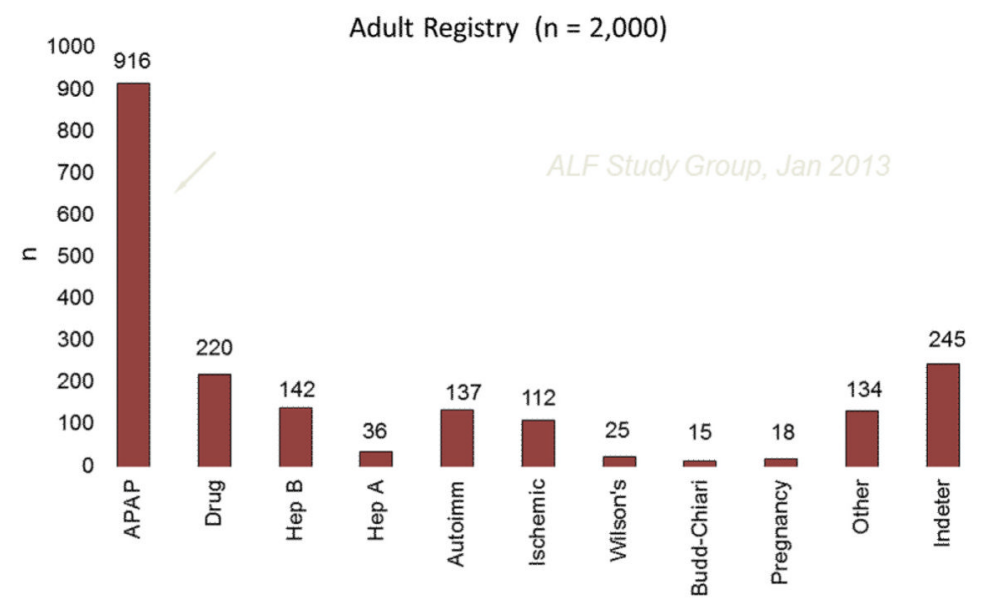

Fig. 1.

Cause of acute liver failure in the United States. 


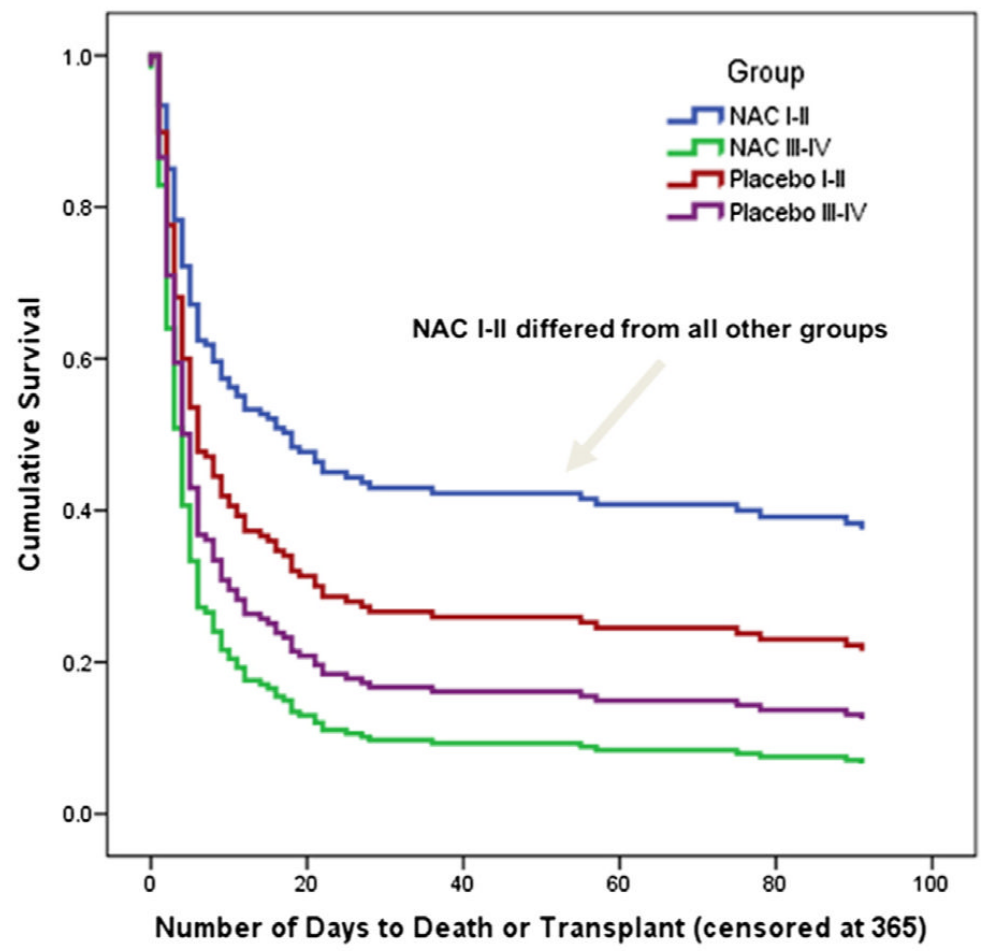

Fig. 2.

Cox proportional hazards model by treatment and coma grade. 
Table 1

Clinical features of different ALF etiology groups $(\mathrm{N}=2000)$

\begin{tabular}{llllll}
\hline & $\begin{array}{l}\text { APAP } \\
(\mathbf{N}=\mathbf{9 1 6})\end{array}$ & $\begin{array}{l}\text { Drug } \\
(\mathbf{N}=\mathbf{2 2 0})\end{array}$ & $\begin{array}{l}\text { Indeterminate } \\
(\mathbf{N}=\mathbf{2 4 5})\end{array}$ & $\begin{array}{l}\text { HepA/HepB } \\
(\mathbf{N = 3 6 / 1 4 2})\end{array}$ & $\begin{array}{l}\text { All Others } \\
(\mathbf{N}=\mathbf{4 4 1})\end{array}$ \\
\hline Age (median) & 37 & 46 & 39 & $49 / 43$ & 45 \\
\hline Gender (\% female) & 76 & 69 & 59 & $44 / 44$ & 71 \\
\hline Jaundice (days) & 1 & 11.5 & 11 & $4 / 8$ & 7 \\
\hline Coma $\geq 3(\%)$ & 53 & 35 & 48 & $56 / 52$ & 38 \\
\hline $\begin{array}{l}\text { Alanine } \\
\text { aminotransferase }\end{array}$ & 3773 & 639.5 & 865 & $2275 / 1649$ & 681 \\
\hline Bilirubin (median) & 4.3 & 19.8 & 21.1 & $12.3 / 18.4$ & 13.9 \\
\hline Tx (\%) & 9 & 40 & 42 & $33 / 39$ & 32 \\
\hline $\begin{array}{c}\text { Spontaneous } \\
\text { survival (\%) }\end{array}$ & 63 & 24 & 22 & $50 / 21$ & 31 \\
\hline Overall survival (\%) & 70 & 58 & 60 & $72 / 55$ & 58 \\
\hline
\end{tabular}


Table 2

NAC results by etiology

\begin{tabular}{|c|c|c|c|c|}
\hline & \multicolumn{2}{|c|}{ Overall Survival } & \multicolumn{2}{|c|}{$\underline{\text { Transplant-Free Survival }}$} \\
\hline & PLB & NAC & PLB & NAC \\
\hline DILI & $17 / 26$ & $15 / 19$ & $7 / 26$ & $11 / 19$ \\
\hline $\mathrm{N}=45$ & $65 \%$ & $79 \%$ & $27 \%$ & $58 \%$ \\
\hline AIH & $10 / 15$ & $7 / 11$ & $4 / 15$ & $1 / 11$ \\
\hline $\mathrm{N}=26$ & $67 \%$ & $64 \%$ & $27 \%$ & $9 \%$ \\
\hline HBV & $6 / 12$ & $19 / 25$ & $2 / 12$ & $10 / 25$ \\
\hline$N=37$ & $50 \%$ & $76 \%$ & $17 \%$ & $40 \%$ \\
\hline Indeterminate & $18 / 26$ & $9 / 15$ & $6 / 26$ & $6 / 15$ \\
\hline $\mathrm{N}=41$ & $69 \%$ & $60 \%$ & $23 \%$ & $40 \%$ \\
\hline
\end{tabular}

Abbreviations: AIH, autoimmune hepatitis; HBV, hepatitis B virus. 